\title{
Overcoming barriers to a diagnosis of dementia: Can we do it?
}

\section{Commentary for 'Persistent barriers and facilitators to seeking help for a dementia diagnosis. A systematic review of $\mathbf{3 0}$ years of the perspectives of carers and people with dementia' by Parker et al}

Marie Poole, Louise Robinson, Jane Wilcock, Greta Rait, Henry Brodaty

Dr Marie Poole PhD, MSc, BA, Research Associate, Population Health Sciences Institute, Newcastle University, Newcastle, UK - Marie.Poole@newcastle.ac.uk

Ms Jane Wilcock MA(Hons.) MSc, Senior Research Fellow, Department of Primary Care \& Population Health, University College London, London, United Kingdom - j.wilcock@ucl.ac.uk

Prof Greta Rait MSc; MD, MRCGP, Clinical Professor of Primary Care and Health Services Research, Department of Primary Care \& Population Health, University College London, London, UK g.rait@ucl.ac.uk

Prof Louise Robinson DBE, MD FRCGP, Professor of Primary Care and Ageing, Population Health Sciences Institute, Newcastle University, Newcastle Upon Tyne, UK -

a.l.robinson@newcastle.ac.uk

Prof Henry Brodaty MBBS, MD, DSc, FRACP, FRANZCP, FAHMS

Scientia Professor of Ageing and Mental Health and Director of the Dementia Collaborative Research Centre, Centre for Healthy Brain Ageing, University of New South Wales, Sydney, Australia h.brodaty@unsw.edu.au

Seeking a diagnosis of dementia is a complex process, contingent on many factors. A person's experiences, views, beliefs and feelings mixed; with the perspectives of other people affect perceptions of dementia. Attitudes and expertise of health care practitioners; as well as cultural and societal understandings and explanations of the condition influence the diagnostic process. That post diagnostic care is often inadequate [1] underscores the importance of access to appropriate systems and resources of support.

As there is still no cure for dementia, the prospect of a diagnosis may seem futile - and more of a life sentence than a positive step. Yet, diagnosis can open the door to support and maintenance of quality of life for persons living with dementia and their families. Encouraging someone to seek a diagnosis of dementia continues to be a priority focus at national and international policy levels [2]. On a global scale, goals for dementia diagnosis rates continue to fall short of goals and recommendations. While in England, diagnosis is estimated to occur in around $68.2 \%$ for people over 65 , above the national target of $66.7 \%$ [3], globally most people living with dementia do not receive a formal diagnosis. Rates of formal diagnoses are only $20-50 \%$ in high income countries, and much lower in lower middle income countries; resulting in a 'treatment gap' due to a lack of access to care and treatment [4]. This may occur for multiple reasons including a lack of knowledge of dementia symptoms, lack of diagnostic services, unclear pathways to diagnosis and inadequate support services post-diagnosis; the barriers facing individuals to seek help for a diagnosis remain key. 
Questions remain over whether people experiencing symptoms, those close to them and health and social care professionals understand and recognise changes and symptoms associated with dementia; and also whether obtaining a diagnosis is important. Common myths about what dementia is (and is not) persist. In Ireland, the "Dementia: Understand Together" campaign revealed that although one in two people reported knowing someone with dementia, only one in four felt that they understood the condition [5]. Alongside understanding and awareness of the condition it is key to understand what prompts or prohibits people from seeking a diagnosis, and what can be done to ensure people are best supported to seek a dementia diagnosis.

National dementia plans and clinical guidance from many countries, and guidelines from international organisations urge practitioners to remain vigilant to patients who display signs and symptoms of dementia and advise on how to achieve an early or timely diagnosis [6, 7]. National and international public awareness campaigns such as World Alzheimer's Day, national dementia awareness days in many countries, and Dementia Friends aim to reduce stigma and fear of dementia, and encourage people to accept and understand the impact of dementia on individuals.

Many countries, including the UK, have national guidelines for practitioners which set out the process of conducting assessments and tests which lead to a formal syndromal and specific disease diagnosis of dementia [8]. A structured approach is useful in achieving a standardised process, however the step prior to engaging with this system is central to obtaining a diagnosis. What are the factors which inhibit or encourage people to seek a diagnosis of dementia?

In their systematic review of studies exploring barriers and facilitators to seeking help for a dementia diagnosis, Parker et al [9]identified myriad and interconnected barriers which prohibit people living with dementia and those who support them from seeking a diagnosis. Denial, fear, stigma, a lack of knowledge of the condition, normalisation, the desire to preserve autonomy, a lack of awareness of changes, inadequate or insufficient informal support, and difficulties faced by carers all play a part in presenting challenges to diagnosis - with many people facing multiple barriers simultaneously. More positively, their identification of facilitators as a way to challenge these persistent barriers relies on recognising symptoms of dementia as a problem - rather than 'normalising' these as part of the ageing process, alongside the importance of a supportive informal network and some prior knowledge of dementia and connections or contacts with healthcare services.

Parker et al emphasised the connections between barriers and facilitators, and how facing several barriers combined to compound issues, requiring multiple facilitators to counter those barriers. It is easy to comprehend how a lack of knowledge of how dementia affects individuals, coupled with a misunderstanding of signs and symptoms continues to present a significant challenge in reducing the fear and stigma of dementia and, may impact on motivations to seek help. This may be experienced in conjunction with practitioners who are unfamiliar with the aspects of the condition and which services can offer diagnostics and post diagnostic support.

The barriers and facilitators identified by Parker et al are complementary to issues raised in the systematic review of Robinson et al, which explored the views of people living with dementia and family carers about the transition to dementia through diagnosis [10]. Reasons for and against diagnostic disclosure; the process of disclosing and receiving a diagnosis; information provision; factors influencing disclosure and recall of a diagnosis; and the impacts of disclosure from the perspectives of the person living with dementia and carers were explored. Findings indicated that although coming to terms with multiple areas of loss is a key challenge for people living with dementia; in general, most people wanted to know their diagnosis, with no apparent long-term negative effect on their psychological health. For family carers, increased responsibilities and 
becoming the main decision-maker were the main areas of adjustment following diagnosis. Taken together, the findings from these reviews demonstrate the challenges of the diagnostic process and post diagnostic support, and present potential ways to address them.

To reduce barriers to seeking help for a dementia diagnosis, Parker et al suggest multi-faceted interventions including those which reduce stigma and empower people with dementia and those who support them such as the development of dementia friendly communities. The COGNISANCE programme [11] is a new international project which may play a part in providing more empirical evidence to improve the experience of diagnosis and post diagnostic support. Five countries Australia, UK, Netherlands, Canada and Poland - are working together with people living with dementia, family carers, a range of health and social care professionals and international organisations to co-design an international toolkit, which seeks to improve the diagnostic process and post diagnostic support. The first stages involve understanding and comparing the current experiences of the diagnostic process and post diagnostic support offered in each partner country. Generic toolkits will be developed and then tailored to regional needs. Finally, the toolkit will be implemented with social marketing campaigns and evaluated for international dissemination.

In summary, over the last 30 years, some progress has been made around obtaining a dementia diagnosis but less with post-diagnostic support. Much still needs to be done not just in low and middle income countries which have the lowest rates of diagnosis, but also in higher income countries. Significant, but not insurmountable challenges still exist. For example, Parker et al highlight that some countries have no word to describe dementia. In Mandarin Chinese, the literal translation is based on two characters which mean "crazy" and "catatonic" [12] or other translations from Chinese meaning "stupid, demented elderly" [13]. In Indian languages, words used for dementia include "childishness", "weak brain" or "tired brain" [14]. In sub-Saharan Africa dementia is described in a way that perpetuates fear and stigma [15]. In a range of cultures and languages, negative words or phrases such as "evaporation" and "second childhood", perpetuate a lack of understanding of the condition and stigma [16]. Encouragingly, emerging changes in understanding and language and new definitions may more accurately reflect how the condition affects people [17].

Barriers to dementia diagnosis and post-diagnostic support remain complex but may be overcome with a better understanding of these barriers and development of facilitators. Continued positive policy, further research, increasing public awareness of dementia and improvements in support services may all play a role in challenging and reducing barriers to achieving a satisfactory dementia diagnostic process and post-diagnostic care.

1. Wells, C.E. and S.J. Smith, Diagnostic care pathways in dementia: A review of the involvement of primary care in practice and innovation. Journal of primary care \& community health, 2017. 8(2): p. 103-111.

2. Alzheimer's Disease International publication team, From plan to impact: Progress towards targets of the global action plan on dementia. 2018.

3. NHS Digital. NHS Digital Dementia Publication. 2020 November 2019 10.01.2020]; Available from:

https://app.powerbi.com/view?r=eyJrljoiMThjZmVhMDYtODYyYSO0OGE3LWEzMWItM2IzYjc 2ODFiMiZlliwidCI6ljUwZjYwNzFmLWJiZmUtNDAxYS04ODAzLTY3Mzc0OGU2MjIIMilsImMiOjh 9.

4. Alzheimer's Disease International. Dementia Statistics. 2020 10.01.2020]; Available from: https://www.alz.co.uk/research/statistics. 
5. Irish Times. Separating fact from fiction: 7 myths about dementia. 22.02.2018 14.01.2020]; Available from: https://www.irishtimes.com/life-and-style/health-family/separating-factfrom-fiction-7-myths-about-dementia-1.3401965.

6. Chow, S., et al., National dementia strategies: what should Canada learn? Canadian Geriatrics Journal, 2018. 21(2): p. 173.

7. Ngo, J. and J.M. Holroyd-Leduc, Systematic review of recent dementia practice guidelines. Age and ageing, 2014. 44(1): p. 25-33.

8. National Institute for Health and Care Excellence. Dementia - assessment, management and support for people living with dementia and their carers. 2018 2.5.2018]; Available from: https://www.nice.org.uk/guidance/indevelopment/gid-cgwave0792/consultation/htmlcontent-3.

9. Parker, M., et al., Persistent barriers and facilitators to seeking help for a dementia diagnosis. A systematic review of 30 years of the perspectives of carers and people with dementia. International Psychogeriatrics, 2020. In press.

10. Robinson, L., et al., The transition to dementia - individual and family experiences of receiving a diagnosis: a review. International Psychogeriatrics, 2011. 23(7): p. 1026-1043.

11. COGNISANCE Project. COGNISANCE: Co-Designing Dementia Diagnosis And Post Diagnostic Care, 2018 10.01.2020]; Available from: https://cheba.unsw.edu.au/consortia/cognisance.

12. Dong, $\mathrm{X}$. and E.-s. Chang, Lost in translation: To our Chinese patient, Alzheimer's meant 'crazy and catatonic'. Health Affairs, 2014. 33(4): p. 712-715.

13. Chen, Z., et al., Challenges of dementia care in China. Geriatrics, 2017. 2(1): p. 7.

14. Dementia Care Notes. What is dementia? 2019 13.06.2019 14.01.2020]; Available from: https://dementiacarenotes.in/dementia/what-is-dementia/.

15. Guerchet, M., et al., Dementia in sub-Saharan Africa: challenges and opportunities, in Alzheimer's Disease International. 2017: London.

16. Berisic, M. and N. Nesvadba, Perceptions of dementia in ethnic communities. 2008, Alzheimer's Australia: Victoria.

17. Central and North West London NHS Foundation Trust. We provide new Chinese word for Dementia. 2020 15.01.2020]; Available from: https://www.cnwl.nhs.uk/news/provide-newchinese-word-dementia/. 